\title{
Management of Nonsustained Ventricular Tachycardia Guided By Electrophysiological Testing
}

\author{
ALAN KADISH, STEPHEN SCHMALTZ, HUGH CALKINS, and \\ FRED MORADY
}

From the Division of Cardiology, Department of Internal Medicine, University of Michigan

Medical Center, Ann Arbor, Michigan

\begin{abstract}
KADISH, A., ET AL.: Management of Nonsustained Ventricular Tachycardia Guided By Electrophysiological Testing. Two hundred eighty patients with spontaneous nonsustained ventricular tachycardia were treated based on the results of electrophysiological testing. Seventy-nine patients had no evidence of structural heart disease, 134 had coronary artery disease, 43 had idiopathic dilated cardiomyopathy, and 24 patients had miscellaneous types of heart disease. Sustained monomorphic ventricular tachycardia was induced during electrophysiological testing in the drug free state in 52 of 280 patients (19\%). Ventricular tachycardia was induced more frequently in patients with coronary artery disease (32\%) than in any of the other groups $(\mathrm{P}<0.001)$. The patients with inducible sustained monomorphic ventricular tachycardia underwent a mean of $1.9 \pm 1.3$ drug trials. Twenty-five patients had the induction of ventricular tachycardia suppressed by pharmacological therapy and were treated with the drug judged to be effective during electropharmacological testing. Twenty-seven patients continued to have inducible sustained monomorphic ventricular tachycardia despite antiarrhythmic therapy and were discharged on the drug that made induced ventricular tachycardia best tolerated. Forty-five of 280 patients (16.1\%) died during a mean follow-up period of $19.6 \pm 14.4$ months. There were 15 sudden cardiac deaths, 21 nonsudden cardiac deaths, 6 noncardiac deaths, and 3 deaths that could not be classified. Sudden cardiac death mortality was lowest in the patients without structural heart disease ( $0 \%$ at 2 years), intermediate in the patients with coronary artery disease and miscellaneous heart disease ( $4 \%$ at 2 years), and highest in the patients with idiopathic dilated cardiomyopathy $(13 \%$ at 2 years; $P<0.01$ for pairwise comparisons). No patient treated with a drug that had suppressed the induction of sustained ventricular tachycardia died suddenly during the follow-up period whereas four of 27 patients who were discharged on "ineffective antiarrhythmic drugs" and 11 of 228 patients without inducible sustained ventricular tachycardia experienced sudden cardiac death during the follow-up period. By multivariate analysis, ejection fraction and inducible ventricular tachycardia during the predischarge electrophysiological test were independent predictors of sudden cardiac death. In conclusion, in patients with spontaneous nonsustained ventricular tachycardia: (1) Arrhythmia inducibility varies depending on the underlying heart disease. Ventricular tachycardia is most often inducible in patients with coronary artery disease and least often in patients without structural heart disease; (2) With the exception of patients with idiopathic dilated cardiomyopathy, management of patients with nonsustained ventricular tachycardia guided by electrophysiological testing appears to result in a low incidence of sudden cardiac death although effects on total mortality are less impressive; and (3) Patients with idiopathic dilated cardiomyopathy and patients with other heart diseases who continue to have inducible ventricular tachycardia despite antiarrhythmic drug therapy are at substantial risk of sudden cardiac death. (PACE, Vol. 16, May, Part I 1993)
\end{abstract}

sudden cardiac death, ejection fraction, ventricular tachycardia

Supported in part by Grant 5MO1-RR00042-25 from the National Institute of Health, Bethesda, Maryland. Dr. Kadish is a recipient of an NIH First Award Number HL40667.

Address for reprints: Alan Kadish, M.D., Northwestern Memorial Hospital, Section of Cardiology, Wesley Pavilion, Suite
524, 250 East Superior Street, Chicago, IL 60611. Fax: (312) 908-4753.

Received January 10, 1992; revision September 24, 1992; revision November 6, 1992; accepted November 9, 1992. 


\section{Introduction}

Nonsustained ventricular tachycardia is associated with an adverse prognosis in certain patients. ${ }^{1-3}$ Several studies have examined the role of ejection fraction, Holter monitoring, the signalaveraged electrocardiogram, and electrophysiological testing in defining prognosis in patients with nonsustained ventricular tachycardia. ${ }^{1-6}$ However, the use of these diagnostic tests and the most appropriate form of therapy in patients with various types of heart disease and nonsustained ventricular tachycardia are as yet unclear.

The purpose of this study was to describe the outcome in a large cohort of patients with various types of heart disease who had nonsustained ventricular tachycardia and whose management was guided by electrophysiological testing.

\section{Methods}

\section{Patient Population}

The present investigation was a retrospective study of 280 patients consecutive with asymptomatic, nonsustained ventricular tachycardia who underwent electrophysiological testing in the drug free state. Patients with nonspecific palpitations were not considered symptomatic for the purposes of this study. Patients who had suffered a cardiac arrest or experienced sustained uniform ventricular tachycardia were not entered into the study. Nonsustained ventricular tachycardia was defined as having a duration of three beats to 30 seconds and was documented by ambulatory monitoring (240 patients) or telemetry recordings. Seventynine patients had no evidence of structural heart disease as determined by cardiac catheterization or the combination of exercise testing and twodimensional echocardiography. One hundred thirty-four patients had coronary artery disease documented by cardiac catheterization or the presence of a Q wave infarction, and 43 patients had an idiopathic dilated cardiomyopathy documented by cardiac catheterization. Twenty-four patients had other types of heart disease, primarily valvular heart disease (Table I). One hundred twentyfive of the 134 patients with coronary disease had evidence of prior myocardial infarction. Demographic characteristics of these patients are described in Table I.

\section{Electrophysiological Study Protocol}

Electrophysiological studies were performed while patients were fasting and not sedated. Informed consent was obtained from all patients prior to electrophysiological testing. Antiarrhyth-

Table I.

Demographic Characteristics of the Patient Population

\begin{tabular}{|c|c|c|c|c|c|}
\hline & \multicolumn{4}{|c|}{ Group Number } & \multirow[b]{2}{*}{ All } \\
\hline & 1 & 2 & 3 & 4 & \\
\hline \multirow{5}{*}{$\begin{array}{l}\text { Heart Disease } \\
\text { Number of pts } \\
\text { Mean Age (years) } \\
\text { Gender }\end{array}$} & None & CAD & $\mathrm{CM}$ & Other ${ }^{\star}$ & \\
\hline & 79 & 134 & 43 & 24 & 280 \\
\hline & $52 \pm 15$ & $62 \pm 11$ & $58 \pm 13$ & $54 \pm 16$ & $58 \pm 13$ \\
\hline & 46 male & 118 male & 37 male & 14 male & 215 male \\
\hline & 33 female & 16 female & 6 female & 10 female & 65 female \\
\hline \multirow{5}{*}{$\begin{array}{l}\text { Mean EF } \\
\text { NYHA Class }\end{array}$} & $0.62 \pm 0.06$ & $0.36 \pm 0.15$ & $0.26 \pm 0.14$ & $0.43 \pm 0.19$ & $0.37 \pm 0.17$ \\
\hline & 61 & 45 & 6 & 10 & 122 \\
\hline & 16 & 47 & 15 & 7 & 85 \\
\hline & 2 & 32 & 16 & 4 & 54 \\
\hline & 0 & 10 & 6 & 3 & 19 \\
\hline
\end{tabular}

$\mathrm{CAD}=$ coronary artery disease; $\mathrm{CM}=$ idiopathic dilated cardiomyopathy; $\mathrm{EF}=$ ejection fraction.

* Other Heart Disease: valvular heart disease 13, hypertensive cardiovascular disease 3; idiopathic hypertrophic cardiomyopathy 7; coronary artery spasm 1. 
mic therapy was discontinued at least five halflives before testing. Three quadripolar catheters were inserted into a femoral vein and positioned in the high right atrium, at the right ventricular apex, and in the right ventricular outflow tract. Leads V1, 1, and 3 and the intracardiac electrograms were displayed on an oscilloscope and recorded on a Siemens-Elema Mingograf 7 recorder (Siemens-Elema, Solna, Sweden).

Ventricular stimulation was performed using a programmable stimulator (Bloom Associates, Narbeth, PA, USA). Rectangular stimuli $2 \mathrm{msec}$ in duration at twice diastolic threshold were used. Up to 3 extrastimuli were introduced using at least two basic drive cycle lengths at the right ventricular apex and at the right ventricular outflow tract or upper septum. ${ }^{7}$ Diastole was scanned as previously described and coupling intervals were limited to $200 \mathrm{msec}^{8}{ }^{8}$ The endpoint of the stimulation protocol was the induction of sustained monomorphic ventricular tachycardia. Nonsustained polymorphic ventricular tachycardia was not an endpoint of the stimulation protocol. If sustained polymorphic ventricular tachycardia or ventricular fibrillation was induced once, stimulation generally was continued in an attempt to induce sustained monomorphic ventricular tachycardia except if multiple countershocks were required to terminate ventricular fibrillation or if the patient refused further testing. ${ }^{9}$ Only sustained monomorphic ventricular tachycardia was considered a positive result.

If sustained monomorphic ventricular tachycardia was induced during the baseline electrophysiological test, the patient underwent serial drug testing in an attempt to suppress the induction of ventricular tachycardia. Sustained monomorphic ventricular tachycardia was also the endpoint used for assessing drug efficacy. If the induction of ventricular tachycardia was suppressed by an antiarrhythmic agent, the patient was treated long term with that drug. If the induction of ventricular tachycardia was not suppressed by antiarrhythmic therapy, patients were treated with the drug that made induced ventricular tachycardia best tolerated, except for two patients who refused therapy. Tolerance of ventricular tachycardia was determined by blood pressure and/or symptoms during sustained ventricular tachycardia. All patients who failed drug testing (except one who re- fused further testing) had undergone at least two electrophysiological tests on different antiarrhythmic drugs.

The following definitions were used with regard to the electrophysiological study: Sustained ventricular tachycardia was defined as lasting > 30 seconds or requiring termination because of hemodynamic compromise. Nonsustained ventricular tachycardia was defined as lasting from three beats to 30 seconds. Monomorphic ventricular tachycardia had a stable QRS morphology when viewed simultaneously in three leads. Polymorphic ventricular tachycardia was defined as having a variable QRS morphology.

\section{Patient Follow-Up}

Follow-up information was obtained from the patient, patient's family, and/or the patient's physician. Eleven $(3.8 \%)$ patients who met the inclusion criteria were lost to follow-up and the remaining 280 patients form the study population. Information was obtained on the occurrence of sustained ventricular tachycardia or cardiac arrest and on the causes of death.

Deaths were classified as sudden cardiac death, other cardiac death, and noncardiac death. For the purposes of this study, sudden cardiac death was defined as instantaneous death or unwitnessed death with no evidence of a nonarrhythmic cause of death. Patients who experienced premonitory symptoms consistent with an arrhythmia, e.g., palpitations or presyncope, were also classified as having sudden cardiac death. The presence of other premonitory symptoms such as chest pain or shortness of breath led to a classification of "other cardiac death." In three patients, insufficient information was available to classify the cause of death. In the analysis of sudden cardiac death and ventricular tachycardia occurrence, these three patients were censored at the time of death.

\section{Statistical Methods}

Data are expressed as mean \pm 1 standard deviation. Frequencies were compared by Chisquare analysis. Survival curves were constructed using the Kaplan-Meier product limit estimate. ${ }^{10}$ The standard errors of survival curves were calculated using the methods of Kaplan-Meier and mul- 
tiple comparisons between curves were performed using the Bonferroni correction method. ${ }^{10} \mathrm{~A} \mathrm{P}$ value of $<0.05$ was considered significant. In examining outcome, the three response variables used were the time until sudden cardiac death, time until death (all causes), and the time until sustained ventricular tachycardia/ventricular fibrillation. Sustained ventricular tachycardia/ventricular fibrillation occurrence was defined as the occurrence of documented sustained ventricular tachycardia or sudden cardiac death. The main goal of the statistical analysis was to assess the dependence of the three response variables on a number of explanatory variables. These explanatory variables are listed in Table II. The statistical method used to assess the effect of these explanatory variables on survival was Cox regression analysis. In the first stage of the analysis, each of the explanatory variables was used to assess its individual effect on survival. Explanatory variables then were combined using a forward selection procedure to determine the best set of variables related to survival. Because survival times differ depending on the underlying heart disease, the analysis was stratified by heart disease. The data were also separately analyzed for the coronary artery disease and cardiomyopathy groups. A P value < 0.10 was considered significant for the purpose of the Cox regression analysis. ${ }^{10}$

Instead of selecting a predefined ejection fraction to separate low risk and high risk groups, logistic regression was used to estimate the probability of death as a function of this variable. ${ }^{10}$ Using

\section{Table II.}

Variables Used in Multivariate Analysis

Age
Sex
Ejection Fraction
NYHA Classification
Prior anti-arrhythmic drug therapy
Results of Baseline electrophysiological testing
Results of Predischarge electrophysiological testing
Anti-arrhythmic drug therapy after discharge
Digoxin therapy
Diurectic therapy
Beta blocker therapy

this logistic regression, the sensitivity and specificity were calculated for different cutoffs of the predicted probabilities. These predicted probabilities were then inverted to yield the resulting cutoffs for the ejection fraction.

\section{Results}

\section{Electrophysiological Testing}

Among the 280 patients who underwent a baseline electrophysiological test, $52(19 \%)$ had inducible sustained monomorphic ventricular tachycardia. The mean cycle length of monomorphic ventricular tachycardia induced during the baseline electrophysiological test was $286 \pm 59$ msec. A mean of $2.3 \pm 0.6$ extrastimuli were required to induce sustained monomorphic ventricular tachycardia. The inducibility of ventricular tachycardia based on the type of underlying heart disease is shown in Figure 1. Ventricular tachycardia was induced more frequently in patients with coronary artery disease $(32 \%)$ than in the other groups $(\mathrm{P}<0.001)$. The induction of ventricular tachycardia in patients with coronary artery disease was dependent upon the level of left ventricular function. Nine of $50(18 \%)$ patients with a left ventricular ejection fraction of $>0.4$ had inducible ventricular tachycardia versus 34 of $84(40 \%)$ patients with a left ventricular ejection fraction < 0.4 ( $\mathrm{P}<0.05)$. Ventricular tachycardia also was induced more frequently in the patients with idiopathic dilated cardiomyopathy (14\%) and with miscellaneous heart diseases $(13 \%)$ than in those without structural heart disease $(0 \% ; \mathrm{P}<0.01$ for both comparisons). The mean cycle length of induced ventricular tachycardia was similar in the various heart disease groups: $285 \pm 59 \mathrm{msec}$ in the patients with coronary artery disease, $297 \pm$ $65 \mathrm{msec}$ in the patients with idiopathic dilated cardiomyopathy, and $280 \pm 71 \mathrm{msec}$ in the patients with miscellaneous heart disease. The mean number of extrastimuli required to induce ventricular tachycardia was also similar regardless of the underlying heart disease.

Among the 228 patients considered to have negative electrophysiological tests, 156 patients had no arrhythmia induced, 59 had nonsustained ventricular tachycardia induced, and 13 had ventricular fibrillation induced. 


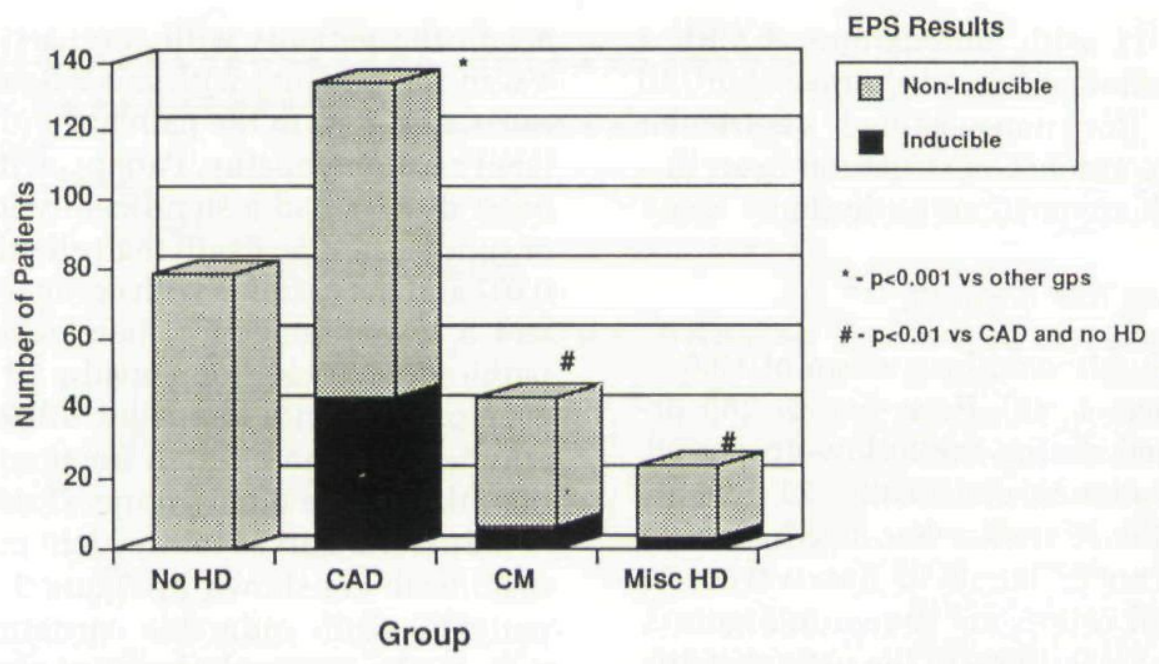

Figure 1. Inducibility of ventricular tachycardia according to type of underlying heart disease. The number of patients with inducible monomorphic ventricular tachycardia during a baseline electrophysiological test is shown in the solid areas and the number of patients without inducible sustained ventricular tachycardia in the shaded areas. Inducibility of ventricular tachycardia was highest in the patients with coronary artery disease (CAD), intermediate in those with idiopathic dilated cardiomyopathy (CM) and miscellaneous heart disease (Misc HD), and lowest in those without structural heart disease (No HD) ( $\mathrm{P}<0.01$ for pairwise comparisons).

\section{Electropharmacological Testing}

The patients with inducible sustained monomorphic ventricular tachycardia underwent a mean of $1.9 \pm 1.3$ drug trials (range $1-5$ ). Three patients refused electrophysiological testing before having failed at least two antiarrhythmic drug trials. They were discharged on empiric therapy (one patient) or no therapy (two patients) and were considered to have persistently inducible ventricular tachycardia for further analysis. None of these three patients died suddenly during the follow-up period. No patient underwent implantation of an implantable cardioverter defibrillator or had arrhythmia surgery performed.

Among 52 patients who underwent electropharmacological testing, 27 continued to have inducible sustained monomorphic ventricular tachycardia and 25 did not. During the baseline electrophysiological test, the mean cycle length of induced ventricular tachycardia ( $279 \pm 59$ vs 292 $\pm 59 \mathrm{msec}$ ) and the mean number of extrastimuli needed to induce ventricular tachycardia $(2.2 \pm$ 0.7 vs $2.4 \pm 0.5$ ) were similar in the patients who did and did not respond during drug testing. In the patients with coronary artery disease, five of nine patients with a left ventricular ejection fraction $>0.4$ had the inducibility of ventricular tachycardia suppressed with antiarrhythmia medication. The patients in whom ventricular tachycardia induction was suppressed by an antiarrhythmic drug were treated long term with that drug; the 27 patients who continued to have inducible sustained ventricular tachycardia were discharged on the drug that made the induced ventricular tachycardia slowest and most hemodynamically stable. Among the 52 patients who had inducible sustained monomorphic ventricular tachycardia in the baseline state, two were discharged on no therapy (at the patient's request), 24 received a type IA antiarrhythmic agent, 16 received amiodarone, 6 received a combination of amiodarone and a type I antiarrhythmic agent, and 4 were treated with a type IC antiarrhythmic agent.

Among 228 patients without inducible sustained monomorphic ventricular tachycardia during the baseline electrophysiological test, 89 received empiric antiarrhythmic therapy for nonsustained ventricular tachycardia or for atrial arrhythmias and 139 received no drug therapy. Forty patients were treated with a type IA antiar- 
rhythmic agent, 11 with amiodarone, 3 with a combination of amiodarone and another agent, 10 with verapamil (for nonsustained ventricular tachycardia in the absence of structural heart disease), and 25 with a type IC antiarrhythmic agent.

\section{Outcome}

Patients were followed for a mean of $19.6 \pm$ 14.4 months (range 1-65). Forty-five of 280 patients $(16.1 \%)$ died during the follow-up period. There were 15 sudden cardiac deaths, 21 nonsudden cardiac deaths, 6 noncardiac deaths, and 3 deaths that could not be classified. Survival curves for deaths from all causes are shown in Figure 2. Two-year survival was $100 \%$ in the patients without heart disease, $83 \%$ in the patients with coronary artery disease, $73 \%$ in the patients with miscellaneous heart diseases, and $68 \%$ in the patients with idiopathic dilated cardiomyopathy. The probability of survival was significantly higher in the patients without structural heart disease $(\mathrm{P}<$ 0.01 ) than in all the other groups. In addition, patients with coronary artery disease had a significantly lower mortality than those with idiopathic dilated cardiomyopathy $(\mathrm{P}<0.05)$.

The incidence of sudden cardiac death at 2 years was $0 \%$ in the patients without heart disease,
$5 \%$ in the patients with coronary artery disease, $4 \%$ in the patients with miscellaneous heart diseases, and $23 \%$ in the patients with idiopathic dilated cardiomyopathy. Patients without structural heart disease had a significantly lower incidence of sudden cardiac death than all other groups $(\mathrm{P}<$ 0.01 ) and the patients with coronary artery disease had a lower incidence than patients with idiopathic dilated cardiomyopathy $(\mathrm{P}<0.02)$. However, patients with idiopathic dilated cardiomyopathy had a mean ejection fraction that was lower than that in the other groups (Table I).

Survival curves for freedom from sudden cardiac death are shown in Figure 3. Four of the 52 patients with inducible sustained ventricular tachycardia during the baseline electrophysiological test died suddenly during the follow-up period. The mean cycle length of induced ventricular tachycardia was $305 \pm 86 \mathrm{msec}$ in the four patients who died suddenly and $284 \pm 57 \mathrm{msec}$ in the 48 patients who did not die suddenly (NS). The number of extrastimuli required to induce ventricular tachycardia was also similar in these two groups $(2.2 \pm 0.5$ extrastimuli vs $2.3 \pm 0.6$ extrastimuli).

Twenty-four of 280 patients either died suddenly or had an episode of sustained ventricular tachycardia during follow-up. One had no under-

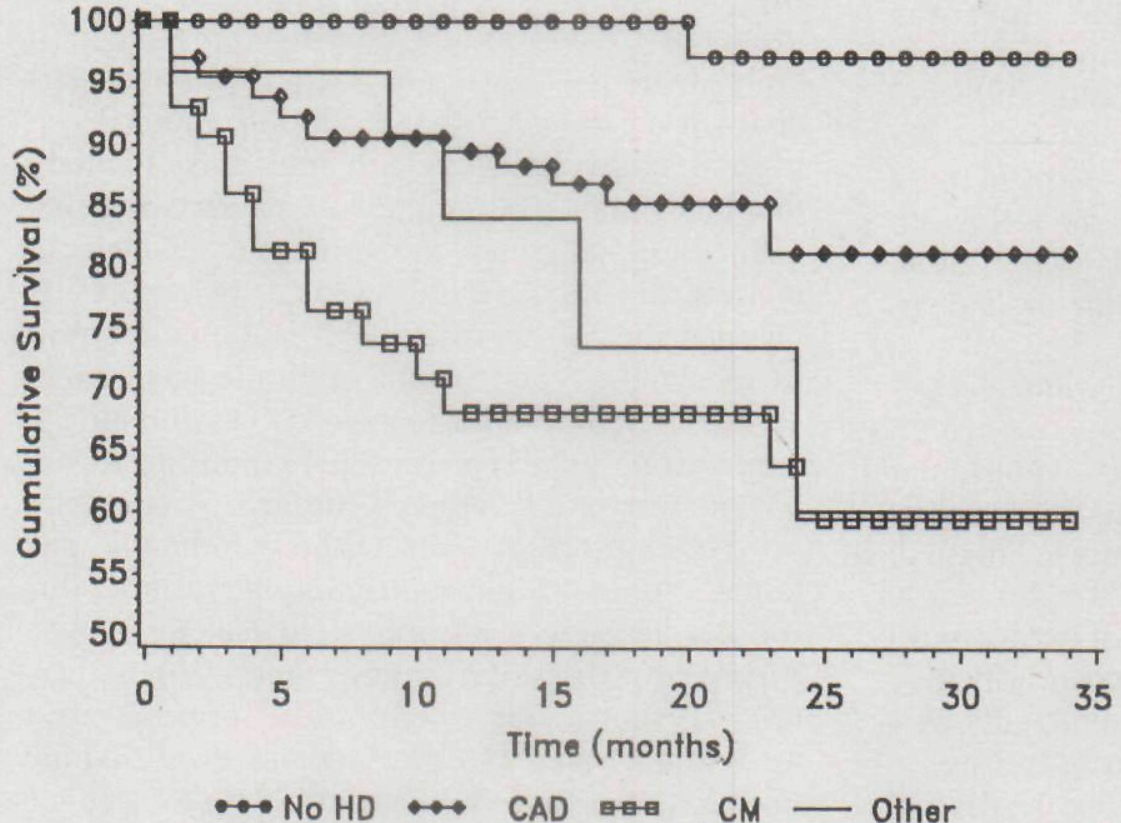

Figure 2. Survival curve analysis of total mortality during follow-up in each of the subgroups. Total mortality was highest in the patients with idiopathic dilated cardiomyopathy, intermediate in those with coronary artery disease and miscellaneous heart disease, and lowest in those without structural heart disease. See text for details. 


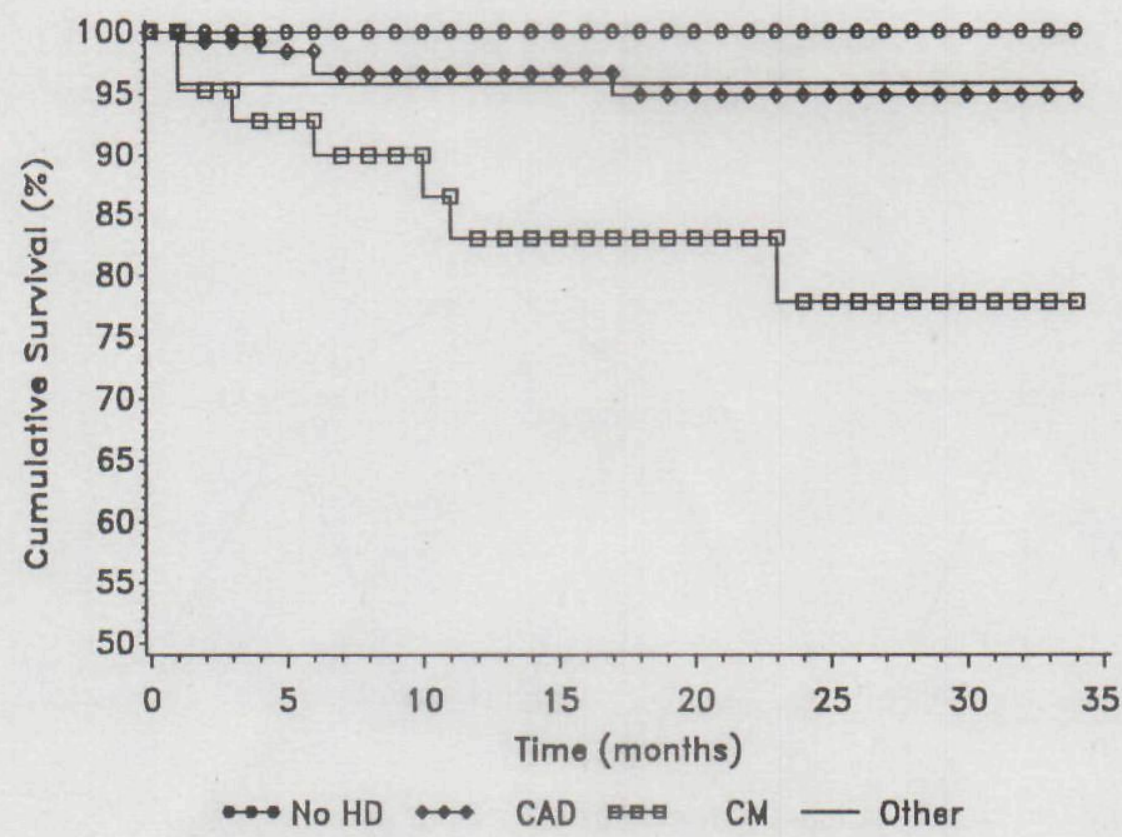

Figure 3. Sudden cardiac death in each of the subgroups. The incidence of sudden cardiac death was highest in patients with idiopathic dilated cardiomyopathy, intermediate in patients with coronary artery diseases and miscellaneous heart disease, and lowest in patients without structural heart disease. See text for details. lying heart disease, 13 had coronary artery disease, 8 had an idiopathic dilated cardiomyopathy, and 2 had miscellaneous heart diseases. Freedom from sustained ventricular arrhythmia occurrence in each of the groups is shown in Figure 4. Patients without structural heart disease were less likely to experience a sustained arrhythmia occurrence during follow-up than were patients with coronary artery disease or idiopathic dilated cardiomyopathy $(\mathrm{P}<0.05)$.

Figures 5 through 7 describe patient outcome based on the results of electrophysiological test-

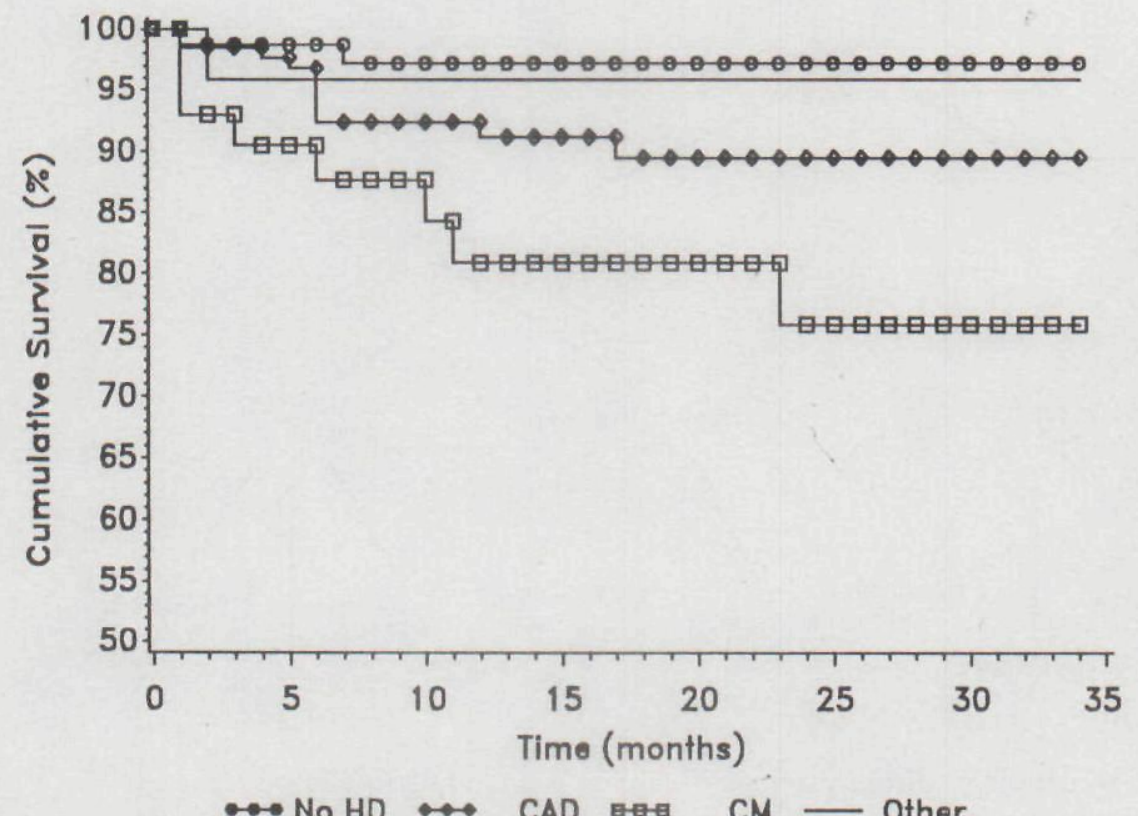

Figure 4. Survival curve analysis of freedom from arrhythmia recurrence in each of the subgroups. Ventricular tachycardia and sudden cardiac death were highest in patients with idiopathic dilated cardiomyopathy, intermediate in those with coronary artery disease and miscellaneous heart diseases, and lowest in those without structural heart disease. See text for details. 


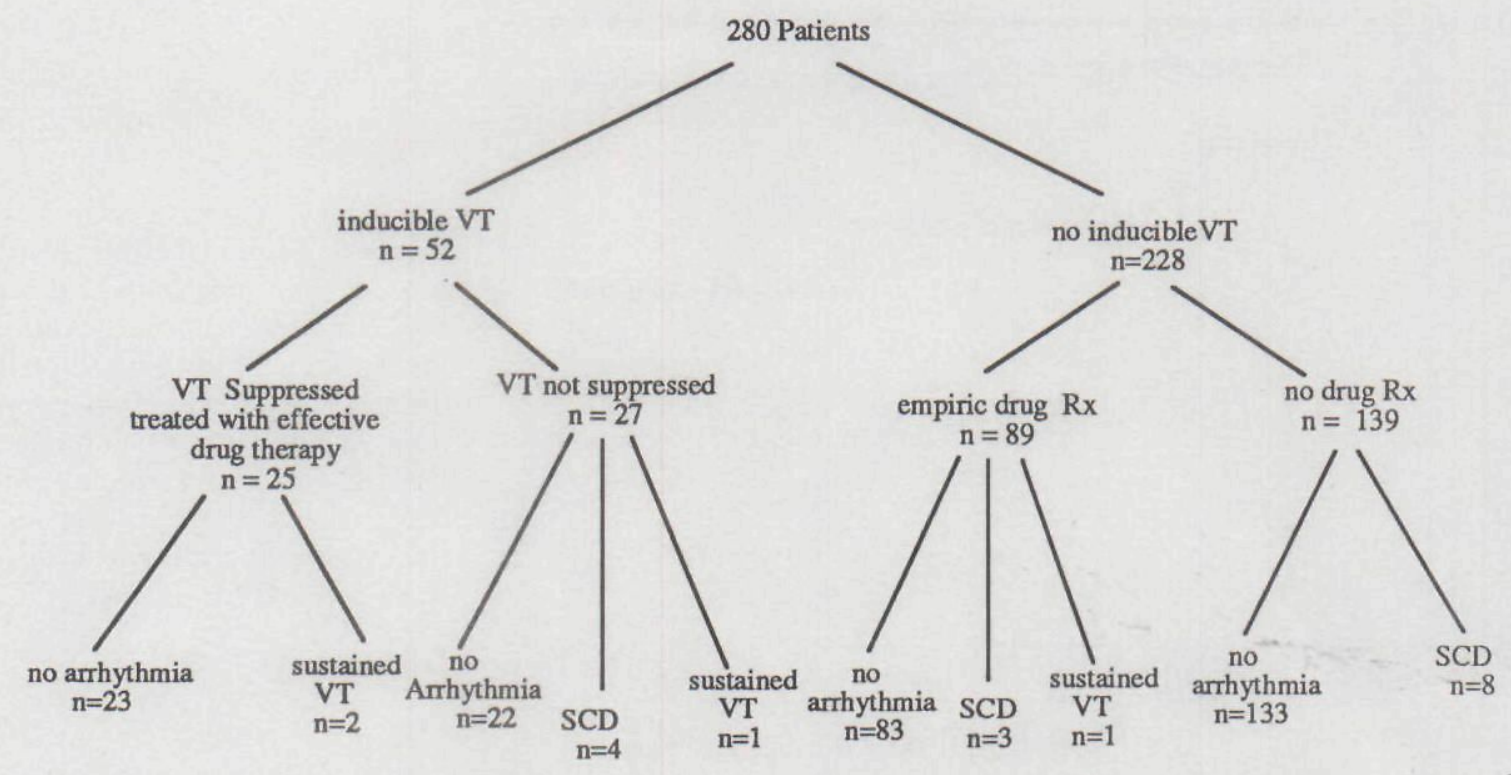

Figure 5. Relationship of results of electrophysiological testing to outcome in the total group of 280 patients.

ing. Frequency data (not actuarial survival) is presented in the figures for simplicity. The relationship between the results of electrophysiological testing and outcome for all patients is shown in Figure 5. Patients who had inducible ventricular tachycardia during the predischarge electrophysi- ological test had the highest actuarial incidence of sudden cardiac death during follow-up $(11 \%$ at 2 years). The incidence of sudden cardiac death at 2 years was $0 \%$ in the patients treated with an antiarrhythmic agent that suppressed the induction of ventricular tachycardia, $4 \%$ in the patients treated
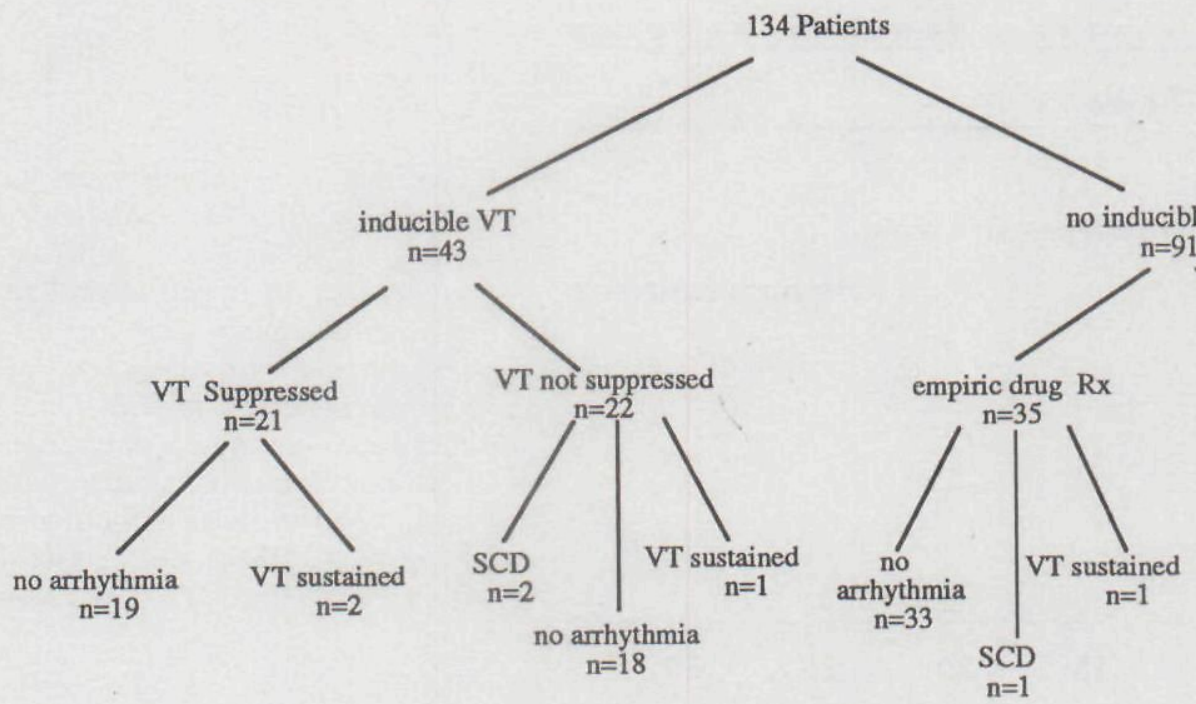

Figure 6. Relationship of the results of electrophysiological testing to outcome in 134 patients with coronary artery disease. 


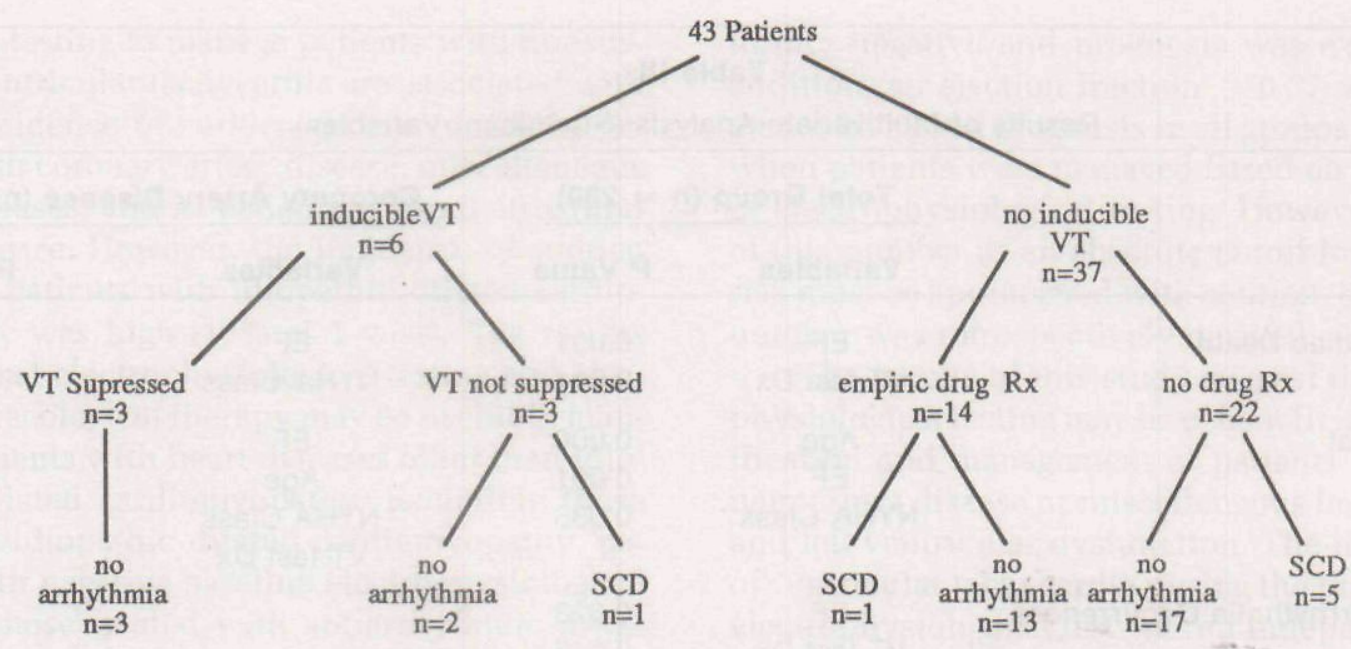

Figure 7. Relationship of the results of electrophysiological testing to outcome in 43 patients with idiopathic dilated cardiomyopathy.

empirically with antiarrhythmic agents despite negative baseline electrophysiological tests, and $5 \%$ in the untreated patients. Data on the 134 patients with coronary artery disease and on the 43 patients with idiopathic dilated cardiomyopathy are shown in Figures 6 and 7. Patients with idiopathic dilated cardiomyopathy were at high risk for sudden cardiac death despite a negative electrophysiological test. If patients with idiopathic dilated cardiomyopathy are excluded from the analysis, the incidence of sudden cardiac death at 1 year was $0 \%$ in the 22 patients who were treated with drugs that were effective in suppressing inducible ventricular tachycardia, $12 \%$ in the 24 patients who did not have inducible ventricular tachycardia suppressed at electrophysiological testing, and $3 \%$ in the 191 patients with a negative baseline electrophysiological test. The incidence of sudden death was not significantly different in those who did ( $2.6 \%$ ) versus did not (3.4\%) receive empiric therapy.

\section{Predictors of Outcome}

Results of the forward selection procedure are shown in Table III.

Using the forward selection procedure, only lower ejection fraction and inducible ventricular tachycardia during the predischarge electrophysiological test correlated with sudden death during follow-up. Older age, lower ejection fraction, and higher New York Heart Association (NYHA) clas- sification all correlated with total mortality during the follow-up period. Sustained ventricular arrhythmia occurrence during the follow-up period was associated with a lower ejection fraction and inducible sustained ventricular tachycardia during the predischarge electrophysiological test.

Survival analysis was repeated in two subgroups: patients with coronary artery disease and patients with idiopathic dilated cardiomyopathy. Despite the high percentage of sudden cardiac death in patients with idiopathic dilated cardiomyopathy, the number of patients was too small to perform the forward selection procedure. In patients with coronary artery disease, increasing age, lower ejection fraction, and higher NYHA classification correlated with sudden cardiac death. Increasing age, higher NYHA classification, and ventricular tachycardia induced during the predischarge electrophysiological test correlated with total mortality. No variables correlated with sustained ventricular arrhythmia occurrence in the patients with coronary artery disease.

Using logistic regression, a left ventricular ejection fraction of 0.37 provided the best discrimination among outcomes (Fig. 8). Patients who had an ejection fraction $>0.37$ had a $0 \%$ incidence of sudden cardiac death at 2 years, whereas those who had an ejection fraction $<0.37$ had a $25 \%$ incidence of sudden cardiac death at 2 years $(\mathrm{P}<$ $0.001)$. In addition, patients with a left ventricular ejection fraction $<0.37$ who had coronary artery 
Table III.

Results of Multivariate Analysis (Significant Variables)

\begin{tabular}{|c|c|c|c|c|}
\hline & \multicolumn{2}{|c|}{ Total Group $(n=280)$} & \multicolumn{2}{|c|}{ Coronary Artery Disease $(n=134)$} \\
\hline & Variables & P Value & Variables & P Value \\
\hline Sudden Cardiac Death & $\begin{array}{c}\text { EF } \\
\text { VT last Dx }\end{array}$ & $\begin{array}{l}0.001 \\
0.011\end{array}$ & $\begin{array}{c}\text { EF } \\
\text { NYHA Class }\end{array}$ & $\begin{array}{l}0.043 \\
0.023\end{array}$ \\
\hline Total Survival & $\begin{array}{c}\text { Age } \\
\text { EF } \\
\text { NYHA Class }\end{array}$ & $\begin{array}{l}0.006 \\
0.001 \\
0.036\end{array}$ & $\begin{array}{c}\text { EF } \\
\text { Age } \\
\text { NYHA Class } \\
\text { VT last Dx }\end{array}$ & $\begin{array}{l}0.013 \\
0.008 \\
0.007 \\
0.094\end{array}$ \\
\hline Sustained Arrhythmia Occurrences & $\begin{array}{c}\text { EF } \\
\text { VT last Dx }\end{array}$ & $\begin{array}{l}0.023 \\
0.046\end{array}$ & & \\
\hline
\end{tabular}

$\mathrm{EF}=$ ejection fraction; NYHA $=$ New York Heart Association Class; VT last $\mathrm{Dx}=$ Inducible VT at predischarge electrophysiological testing.

disease or miscellaneous heart disease and a negative baseline electrophysiological test had a 2-year sudden cardiac death mortality of only $6 \%$.

Survival analysis was repeated to determine the relationship between therapy with specific antiarrhythmic agents and subsequent arrhythmia events. By univariate analysis, therapy with type IA, IC, or III antiarrhythmic agents was not associ- ated with the occurrence of sudden cardiac death or ventricular tachycardia.

\section{Discussion}

\section{Main Findings}

The major finding of this study is that the use of electrophysiological testing and electropharma-

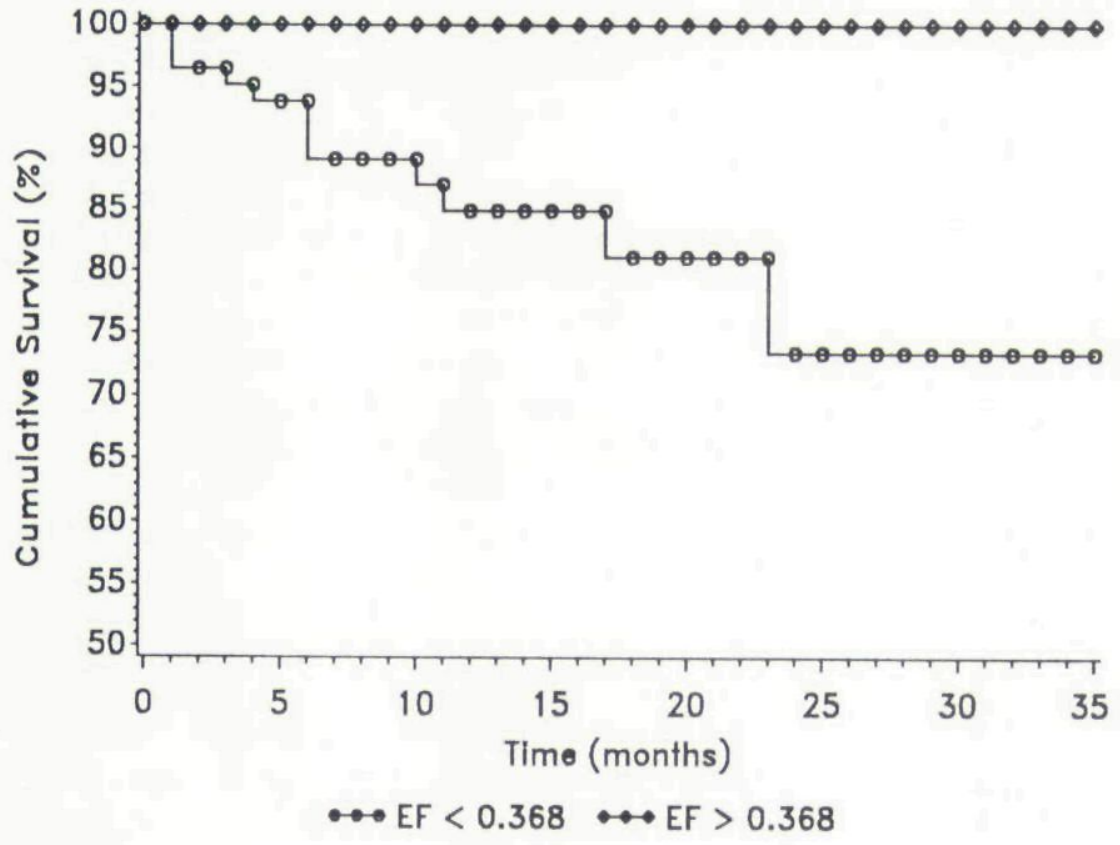

Figure 8. Freedom from sudden cardiac death in the total patient population stratified by ejection fraction (EF). An EF of 0.37 had a sensitivity of $93 \%$ and a specificity of $59 \%$ in predicting sudden cardiac death. 
cological testing to manage patients with nonsustained ventricular tachycardia are associated with a low incidence of sudden cardiac death in patients with coronary artery disease, miscellaneous heart diseases, and in patients without structural heart disease. However, the incidence of sudden death in patients with idiopathic dilated cardiomyopathy was high ( $17 \%$ at 1 year). The results suggest that electrophysiological testing and electropharmacological therapy may be useful in managing patients with heart diseases other than idiopathic dilated cardiomyopathy. Excluding those who had idiopathic dilated cardiomyopathy, patients with negative baseline electrophysiological tests or those treated with antiarrhythmic drugs that suppressed the induction of ventricular tachycardia had a low incidence of sudden cardiac death during follow-up. In contrast, patients with persistently inducible ventricular tachycardia and patients with idiopathic dilated cardiomyopathy remained at substantial risk of sudden cardiac death. However, the results of this study must be regarded as preliminary because of the potential for proarrhythmia in the patients treated empirically and because total mortality was not clearly related to the results of electrophysiological testing.

\section{Effect of Underlying Heart Disease}

Sudden cardiac death and total mortality appeared to be dependent on the underlying heart disease in this study. Patients without structural heart disease had a negligible incidence of ventricular tachycardia or death during follow-up. Patients with coronary artery disease and miscellaneous heart diseases had a moderately low mortality from sudden cardiac death, whereas patients with idiopathic dilated cardiomyopathy had a high mortality from sudden and nonsudden cardiac death. However, patients with idiopathic dilated cardiomyopathy had lower left ventricular ejection fractions than those in other groups and thus the independent risk of the type of underlying heart disease on outcome cannot be established with certainty.

Electrophysiological testing was not of value in patients without structural heart disease who had asymptomatic nonsustained ventricular tachycardia. electrophysiological tests were uni- formly negative and prognosis was excellent. In addition, an ejection fraction $>0.37$ was associated with a good prognosis in all groups of patients when patients were managed based on the results of electrophysiological testing. However, the use of this number as an absolute cutoff for assessing risk must be approached with caution, because the number was retrospectively derived.

The results of this study suggest that electrophysiological testing may be of benefit in risk stratification and management of patients with coronary artery disease or miscellaneous heart disease and left ventricular dysfunction. The inducibility of ventricular tachycardia during the predischarge electrophysiological test was an independent predictor of sudden cardiac death. The value of a negative baseline electrophysiological test in this group of patients cannot be determined with certainty in the absence of a control group not subjected to electrophysiological testing. However, this incidence of sudden death of $2 \%$ per year is lower than in several groups of historical controls with untreated spontaneous nonsustained ventricular tachycardia. ${ }^{1-3}$ When the analysis was confined only to patients with coronary artery disease, the raw incidence of sudden cardiac death was lower in patients with negative predischarge electrophysiological tests than those with positive ones ( $6 \%$ vs $14 \%$ ), but this difference did not reach significance perhaps because of the smaller number of patients. A positive electrophysiological test also appeared to be of value. Patients with a positive baseline electrophysiological test in whom ventricular tachycardia was suppressed by antiarrhythmic therapy had a $0 \%$ incidence of sudden cardiac death whereas those with persistently inducible ventricular tachycardia had a $20 \%$ incidence of sudden cardiac death at 2 years, suggesting that the results of electropharmacological testing are predictive of outcome in these patients. In patients with idiopathic dilated cardiomyopathy, a negative electrophysiological study had no predictive value. Six of 37 patients with a negative electrophysiological test died suddenly during follow-up. However, it is possible that a positive electrophysiological test was useful in these patients. Although none of the three patients with a positive baseline electrophysiological test who had ventricular tachycardia suppressed by antiarrhythmic medications experienced sudden cardiac death or 
ventricular tachycardia during follow-up, this number of patients clearly is too small to allow any conclusions.

\section{Results of Prior Studies}

The results of several prior studies that included relatively small numbers of patients have been divergent. Some studies have suggested that electrophysiological studies are of no value in risk stratification or patient management, while others have suggested that the noninducibility of ventricular tachycardia predicts a low incidence of sudden death. ${ }^{11-13}$ The majority of studies have concluded that the inducibility of sustained ventricular tachycardia portends a poor prognosis. ${ }^{6,14,15}$

Three recent studies of 100 or more patients have examined the value of electrophysiological testing in patients with nonsustained ventricular tachycardia. Hammill et al. ${ }^{16}$ studied 110 patients with a variety of types of heart disease, and followed them for a mean of 15 months. In their study, only the ejection fraction and the presence of congestive heart failure were independent predictors of outcome, and electrophysiological testing was not of major benefit in risk stratification. Kowey et al. ${ }^{17}$ studied 205 asymptomatic patients with coronary disease and remote myocardial infarction. Only left ventricular function correlated significantly with outcome and electrophysiological testing did not appear to be of major value. Unexpectedly, 14 of 45 patients (31\%) had ventricular tachycardia or sudden cardiac death despite receiving therapy guided by electropharmacological testing. ${ }^{17}$ This incidence of ventricular tachycardia and sudden death is inexplicably higher than noted in historical controls who presented with sustained ventricular tachycardia. ${ }^{18}$ Wilber et al. ${ }^{19}$ reported the results of electrophysiological testing in 100 consecutive patients with ejection fractions of $<0.40$, spontaneous nonsustained ventricular tachycardia, and prior myocardial infarction. The persistence of inducible sustained ventricular tachycardia was the only independent predictor of sudden cardiac death in that study.

Prior studies have suggested that electrophysiological testing is not useful in managing patients with idiopathic dilated cardiomyopathy. ${ }^{20,21} \mathrm{~Pa}$ tients with negative electrophysiological tests had a high risk of sudden cardiac death in studies by Kron et al. ${ }^{20}$ and Das et al. ${ }^{21}$ The results of the present study are in agreement with these findings. One potential complicating feature in interpreting the results in patients with dilated cardiomyopathy is that a recent study has suggested that bradycardia may be a common mechanism of sudden cardiac death in such patients. ${ }^{22}$ Thus, diagnostic tests and therapeutic maneuvers directed at tachyarrhythmias may be less beneficial in such patients.

The reasons for the different findings in this study compared to some other recent studies are unclear. The major difference between this study and the study of Kowey et al. ${ }^{17}$ is the low incidence of sudden cardiac death in this study in patients who received antiarrhythmic therapy guided by electrophysiological testing. This low incidence is similar to that described by Klein and Machell ${ }^{15}$ and by Wilber et al. ${ }^{19}$ One potential explanation is that in the multicenter study by Kowey et al., ${ }^{17}$ there may have been differences in the endpoints of electropharmacological testing. The role of electropharmacological testing in the management of patients who present with sustained tachyarrhythmias is also somewhat controversial. Although a large number of studies (reviewed in reference 23) have suggested that suppression of inducible sustained ventricular tachycardia by type I antiarrhythmic drugs portends a good prognosis, recent studies such as those by Poole et al. ${ }^{24}$ suggests that this may not be true. However, in the present study, patients presenting with nonsustained ventricular tachycardia who had inducible ventricular tachycardia suppressed by antiarrhythmic drugs had a $0 \%$ incidence of sudden cardiac death at 2 years.

The findings of this study are generally in agreement with those of Wilber et al. ${ }^{19}$ However, there are two important differences between the two studies aside from the larger number of patients in the present study. Rather than selecting a predefined ejection fraction for inclusion in the study, we analyzed data from all patients and performed logistic regression prospectively to define an ejection fraction (0.37) that provided an excellent separation between patients with and without subsequent cardiac death. In addition, we included all patients regardless of the nature of the underlying heart disease, allowing a comparison among different heart disease groups.

In contrast to the findings of the CAST study, ${ }^{25}$ therapy with antiarrhythmic drugs in gen- 
eral and with type IC antiarrhythmic drugs in particular did not correlate with the occurrence of sudden cardiac death or ventricular tachycardia. One potential explanation is that in the present study these drugs were used empirically only in patients with negative electrophysiological studies. It is possible that patients with inducible ventricular tachycardia are at higher risk of proarrhythmia from type IC antiarrhythmic agents. In addition, a number of patients who received type IC antiarrhythmic agents in this study had normal left ventricular function and this also may have contributed to lowering the risk of a proarrhythmic response to antiarrhythmic drugs.

\section{Limitations}

The major limitation of this study is the absence of an untreated or placebo control group. Therefore, it cannot be proven that management based on the results of electrophysiological testing improved the outcome in patients who had inducible ventricular tachycardia. The response of inducible ventricular tachycardia to antiarrhythmic drugs may itself be a marker of good outcome. Although comparison with historical controls ${ }^{1-3}$ suggests that the incidence of sudden cardiac death in patients in this study who had inducible ventricular tachycardia was low, such retrospective comparisons have major limitations. A second limitation of this study is that 11 patients were lost to follow-up and that the mechanism of deaths could not be determined with certainty in three patients. However, this represents a low percentage of the total number of patients in the study $(3.8 \%)$ and of total deaths $(6.3 \%)$ and thus any error introduced into the study by missing data should be small. In addition, 89 of 228 patients with a negative baseline electrophysiological test were treated with antiarrhythmic drugs by their referring physicians. However, drug therapy was not an independent predictor of subsequent events in the study and the incidence of sudden cardiac death was similar in the patients who received empiric drug therapy and those who did not. Another limitation of this study is the small number of patients in the group with miscellaneous heart disease. In addition, patients in this group contained various diagnoses including hypertrophic cardiomyopathy and valvular heart disease. Although these patients were included in the study so that consecutive patients could be reported, the numbers are probably too small to draw any meaningful conclusions. In addition, we did not analyze the occurrence of sustained ventricular tachycardia alone as a separate outcome but only in combination with cardiac arrest. This was done to limit the number of statistical analyses. Finally, the response rate to antiarrhythmic drugs in this study was unusually high. This may have in part been secondary to the fact that inducible nonsustained ventricular tachycardia $(<30 \mathrm{sec})$ was considered a drug success. This unusually high response rate may limit the applicability of these results to other patient populations.

\section{Clinical Implications}

The results of this study must be regarded as preliminary because of the absence of a control group and because of the small number of patients in some subgroups. However, they suggest that electrophysiological testing may be of benefit in risk stratification and management of some patients with nonsustained ventricular tachycardia depending on left ventricular function and the type of heart disease. Among patients who have left ventricular dysfunction, those with coronary artery disease or miscellaneous heart diseases and a negative electrophysiological test had a low incidence of sudden cardiac death and may not require antiarrhythmic drug therapy. Patients who have a positive baseline electrophysiological test and in whom the induction of ventricular tachycardia is suppressed by antiarrhythmic drugs also had a low incidence of sudden cardiac death, and electropharmacological testing may be a useful guide to therapy. However, patients who have a positive electrophysiological test and in whom the induction of ventricular tachycardia cannot be suppressed by antiarrhythmic drugs have a high incidence of sudden death as do patients with idiopathic dilated cardiomyopathy regardless of the results of electrophysiological testing. These patients therefore may be appropriate candidates for nonpharmacological therapy, such as placement of an implantable cardioverter defibrillator. The risk to benefit ratio of device implantation or other therapy in these types of patients remains to be determined by larger clinical trials using placebo controls. 


\section{References}

1. Bigger JT, Fleiss JL, Kleiger R, et al., and the Multicenter Post-infarction Research Group. The relationships among ventricular arrhythmias, left ventricular dysfunction and mortality in the two years after myocardial infarction. Circulation 1984; 69: 250-258.

2. Mukharji J, Rude FE, Poole K, MILIIS Study Group. Risk factors for sudden death following acute myocardial infarction: Two-year follow-up. Am J Cardiol 1984; 54:31-36.

3. Moss AJ, Davis HT, Decamilla J, et al. Ventricular ectopic beats and their relation to sudden and nonsudden cardiac death after myocardial infarction. Circulation 1979; 60:998-1003.

4. Holmes J, Kubo SH, Cody RJ, et al. Arrhythmias in ischemic and nonischemic dilated cardiomyopathy: Prediction of mortality by ambulatory electrocardiology. Am J Cardiol 1985; 55:166-151.

5. Dennis AR, Cody DV, Young AA, et al. Prognostic significance of ventricular tachycardia and fibrillation induced at programmed stimulation and delayed potentials detected on the signal-averaged electrocardiograms of survivors of acute myocardial infarction. Circulation 1986; 74:731-745.

6. Buxton AE, Marchlinski FE, Flores BT, et al. Nonsustained ventricular tachycardia in patients with coronary artery disease: Role of electrophysiology study. Circulation 1987; 75:1178-1185.

7. Morady F, DiCarlo L, Winston S, et al. A prospective comparison of triple extrastimuli and left ventricular stimulation in studies of ventricular tachycardia induction. Circulation 1984; 70:52-57.

8. Morady F, DiCarlo LA, Baerman J, et al. Comparison of coupling intervals that induce clinical and nonclinical forms of ventricular tachycardia during programmed stimulation. Am J Cardiol 1986; 57:1269-1273.

9. Kou WH, deBuitleir M, Kadish A, et al. Sequelae of nonsustained polymorphic ventricular tachycardia induced during programmed ventricular stimulation. J Am Coll Cardiol 1989; 64: 1148-1151.

10. Kalbfleisch JD, Pretice RL. Statistical Analysis of Failure Time Data. New York, John Wiley \& Sons, Inc., 1980, pp. 70-117.

11. Veltri EP, Platia EV, Griffith LSC, et al. Programmed electrical stimulation and long-term followup in asymptomatic, nonsustained ventricular tachycardia. Am J Cardiol 1985; 56:309-314.

12. Sulpizi AM, Friehling TD, Kowey PR. Value of electrophysiologic testing in patients with nonsustained ventricular tachycardia. Am J Cardiol 1987; 59:841-845

13. Kharsa MH, Gold RL, Moore H, et al. Long-term outcome following programmed electrical stimulation in patients with high-grade ventricular ectopy. PACE 1988; 11:603-609.

14. Gomes JAC, Hariman RI, Kang PS, et al. Pro- grammed electrical stimulation in patients with high grade ventricular ectopy: Electrophysiologic findings and prognosis for survival. Circulation 1984; 70:43-51.

15. Klein RC, Machell C. Use of electrophysiologic testing in patients with nonsustained ventricular tachycardia: Prognostic and therapeutic implications. J Am Coll Cardiol 1989; 111:860-867.

16. Hammill SC, Trusty JM, Wood DL, et al. Influence of ventricular function and presence or absence of coronary artery disease on results of electrophsiologic testing for asymptomatic nonsustained ventricular tachycardia. Am J Cardiol 1990; 65: $722-728$.

17. Kowey PR, Waxman HL, Greenspon A, et al., and the Philadelphia Arrhythmnia Group. Value of electrophysiologic testing in patients with previous myocardial infarction and nonsustained ventricular tachycardia. Am J Cardiol 1990; 65: 594-598.

18. Rae AP, Greenspan AM, Spielman SR, et al. Antiarrhythmic drug efficacy for ventricular tachyarrhythmias associated with coronary artery disease as assessed by electrophysiologic studies. Am J Cardiol 1985; 55:1494-1499.

19. Wilber DJ, Olshansky B, Moran JF, et al. electrophysiological testing and nonsustained ventricular tachycardia: Use and limitations in patients with coronary artery disease and impaired ventricular function. Circulation 1990; 82:350-358.

20. Kron J, Hart M, Schual-Berke S, et al. Idiopathic dilated cardiomyopathy: Role of programmed electrical stimulation and Holter monitoring in predicting those at risk of sudden death. Chest 1988; 93:85-90.

21. Das SK, Morady F, DiCarlo L, et al. Prognostic usefulness of programmed ventricular stimulation in idiopathic dilated cardiomyopathy without symptomatic ventricular arrhythmias. Am J Cardiol 1986; 58:998-1000.

22. Luu M, Stevenson WG, Stevenson LW, et al. Diverse mechanisms of unexpected cardiac arrest in advanced heart failure. Circulation 1989; 80: 1675-1680.

23. Gottlieb C, Josephson ME. Programmed stimulation in the evaluation of life-threatening or potentially life-threatening ventricular arrhythmias. J Cardiovasc Drugs Ther 1987; 1:155-159.

24. Poole JE, Mathisen TL, Kudenchuk PJ, et al. Longterm outcome in patients who survive out of hospital ventricular fibrillation and undergo electrophysiologic studies: Evaluation by electrophysiologic subgroups. J Am Coll Cardiol 1990; 16: 657-665.

25. The Cardiac Arrhythmia Suppression Trial (CAST) Investigators. Effect of encainide and flecainide on mortality in a randomized trial of arrhythmia suppression after myocardial infarction. N Engl J Med 1989; 321:406-412. 
This document is a scanned copy of a printed document. No warranty is given about the accuracy of the copy. Users should refer to the original published version of the material. 